\title{
Utility of quantitative antigen assay for SARS-CoV-2 in the clinical situation Single center experience from Japan
}

Masayuki Nagasawa ( $\sim$ mnagasawa.ped@tmd.ac.jp)

Musashino Red Cross Hospital https://orcid.org/0000-0002-8085-4940

Reiko Inayoshi

Musashino Red Cross Hospital: Musashino Sekijuji Byoin

Yoshimi Yamaguchi

Musashino Red Cross Hospital: Musashino Sekijuji Byoin

Maki Furuya

Musashino Red Cross Hospital: Musashino Sekijuji Byoin

Minoru Dohi

Musashino Red Cross Hospital: Musashino Sekijuji Byoin

Ken Ueda

Musashino Red Cross Hospital: Musashino Sekijuji Byoin

Naoshige Harada

Musashino Red Cross Hospital: Musashino Sekijuji Byoin

Reiko Taki

Musashino Red Cross Hospital: Musashino Sekijuji Byoin

Masayuki Kurosaki

Musashino Red Cross Hospital: Musashino Sekijuji Byoin

\section{Research Article}

Keywords: SARS-CoV-2, COVID-19, quantitative antigen assay, PCR, cut off value, sensitivity

Posted Date: June 2nd, 2021

DOl: https://doi.org/10.21203/rs.3.rs-478817/v1

License: (c) (i) This work is licensed under a Creative Commons Attribution 4.0 International License.

Read Full License 


\section{Abstract}

From December 26, 2020 to February 28, 2021, quantitative antigen test (Ag test) of SARS-CoV-2 was conducted by using a nasopharyngeal swab for 990 patients aged from 0 to 99 years old (average age; $63.1+/-22.5$ years old, male: 537 , female: 463 ), who presented at least one of the following symptoms, such as fever, respiratory symptoms, and taste disorder. In 578 patients, Ag test and PCR test were conducted at the same time and these patients were analyzed. In 74 patients with $\mathrm{Ag}>=1.0,44$ patients were PCR positive (positive ratio $=0.59$ ). In 43 patients with $\mathrm{Ag}>=10.0,41$ patients were PCR positive (positive ratio $=0.95$ ), and all of 42 patients with $\mathrm{Ag}>=100.0$ were $\mathrm{PCR}$ positive. In 504 patients with $\mathrm{Ag}<$ 1.0 , only five patients were PCR positive (positive ratio $=0.01$ ). In 31 patients with $10>A g>=1.0$, three patients were PCR positive (positive ratio $=0.10$ ). All eight patients presented a typical pneumonia, and five patients had a history of close contact with COVID-19 patients within 14 days. With an appropriate cut off value, Ag test is concluded to have enough sensitivity and specificity. Considering the advantage of handiness, rapidness, high-throughput and economy over PCR test, Ag test seems useful diagnostic method for COVID-19 in the pandemic situation.

\section{Introduction}

The acute respiratory infectious disease caused by SARS-CoV-2, named COVID-19, which emerged in December, 2019 at Wuhan in China[1, 2] has spread out around the whole world within several months and the number of the infected people has increased up to approximately 130 million, including the dead person more than 3 million at a point in time in April, 2021 in the whole world[3]. This pandemic infection does not still stand at present.

From November, 2020 through February, 2021, the third wave of infection attacked Japan. In this period, outbreaks in the nursing homes for elderly people and the medical hospitals became the serious problem in addition to the increased number of the infected person, because these patients are at high risk for severe infection. To prevent an outbreak of infection in the nursing homes and the hospital, early detection of infected patients is also important in addition to the universal protective and preventive management to minimize the outbreak. For the early detection of infected patients, sensitive, rapid, easier and less expensive diagnostic tests are being required. In this setting, antigen test for SARS-CoV- 2 is expected to work as an alternative and useful diagnostic method for PCR test[4, 5]. We report our retrospective experience to investigate the sensitivity and specificity of quantitative antigen test for SARSCoV2 (Ag test) provided by SYSMEX (Kobe, Japan) in the real clinical situation.

\section{Methods}

The Musashino Red Cross Hospital is a tertiary center hospital in Tama area, just west of Tokyo, which accepts more than 10,000 ambulances annually, and designated as infection center hospital in this area. We conducted Ag test for the patients with at least one of the following symptoms, such as fever, respiratory symptoms, and taste disorder[6], from December 26, 2020 to February 28, 2021. 


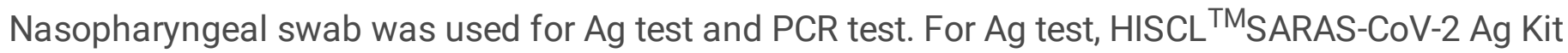
(SYSMEX; Kobe, Japan) was used and assay was carried out automatically by HISCL-5000 (SYSMEX; Kobe, Japan). Briefly, protein was extracted from the nasopharyngeal swab, reacted with biotinylated antiSARS-CoV2 antibody, and then further reacted with streptavidin-bound micro-magnetic beads, which was precipitated, washed, and then reacted with alkaline-phosphatase conjugated secondary anti-SARS-CoV-2 antibody. Sample was washed and then substrate was added and chemiluminescence was measured. Each procedure was performed in accordance to the protocol manual of the supplier, and cut off value > $=1.0$ was determined positive according to a supplier document. Clinical information of each patient was obtained from the electronic medical records, and analyzed with the results of Ag test, PCR test and chest CT scan image. Diagnosis of COVID-19 was principally determined on the basis of positive PCR result.

\section{Results}

From December 26, 2020 to February 28, 2021, quantitative antigen test (Ag test) of SARS-CoV-2 was conducted for 990 patients aged from 0 to 99 years old (average age; $63.1+/-22.5$ years old, male: 537 , female: 463). In 32 patients, Ag test was repeated twice, and all tests were negative. In 407 patients, 243 patients were negative for Ag test, and 164 patients were negative for both of Ag test and PCR test, which was conducted in the sample that was obtained at a different day, usually one to 14 days later from $\mathrm{Ag}$ test. In 578 patients, Ag test and PCR test were conducted at the same time and these patients were analyzed below.

The results were shown in Table 1 and Fig. 1. In 74 patients with $\mathrm{Ag}>=1.0,44$ patients were positive for PCR test (positive ratio $=0.59$ ). In 43 patients with $\mathrm{Ag}>=10.0,41$ patients were PCR positive (positive ratio $=0.95$ ), and all of 42 patients with $A g>=100.0$ were $P C R$ positive. $\ln 504$ patients with $A g<1.0$, only five patients were positive for PCR test (positive ratio $=0.01$ ), all of whom presented typical pneumonia and three had a history of close contact with COVID-19 patients. In 31 patients with $10>\mathrm{Ag}>=1.0$, three patients were positive for PCR test (positive ratio $=0.10$ ), and all of these patients had a typical pneumonia and one had a history of close contact with COVID-19 patients. As the other 28 patients with negative PCR test had no sign of pneumonia in the chest CT scan image, and no history of close contact with COVID-19 patients within 14 days, they were clinically determined not be infected with SARS-CoV-2. For two patients with negative PCR test and $\mathrm{Ag}>=10$, one was 11-year-old boy and had no history of contact with COVID-19 patient with Ag test of 13.8. The other of 64-year-old male with Ag test of 54.7 had a typical pneumonia and his wife was positive for PCR test. The latter patient was clinically managed as COVID-19.

Table 2 presented the cases in which PCR test was positive and $\mathrm{Ag}$ test was negative $(\mathrm{Ag}<1.0)$. All patients presented typical subpleural interstitial pneumoniae in the chest CT scan image, and three out of five had a history of close contact with COVID-19 patients within 14 days.

Figure2 presented the relation of $\mathrm{Ag}$ cut off value and the date when the test sample was obtained in the patients with positive PCR test and $\mathrm{Ag}>=1.0$, whose onset date was determined from the clinical 
information. Five patients were dead (red circle), and five patients received artificial respiratory management and survived (green circle). Although, the sample size was small, there was no apparent correlation with Ag cut off value at the diagnosis and prognosis. Within at least 10 days after the onset, Ag test seemed stable and useful as a diagnostic method for COVID-19.

\section{Discussion}

The advantage of antigen test is the handiness, rapidness, high-throughput and economy. It takes only 15-20 minutes to get the result, a one-third of the common PCR testing, and costs one-third of PCR test in Japanese health insurance system. Furthermore, automated antigen assay by HISCL-5000 (SYSMEX; Kobe, Japan) can manage more samples than PCR test at once.

It has been reported that sensitivity of antigen test is inferior to that of PCR test[7]. However, viral load is much higher between 2 days before and five days after the onset[8], when the infection control management is most important to prevent and minimize an outbreak. In this setting, sensitivity of antigen test is practically enough to investigate the symptomatic patients. It has been reported that $45 \%$ of COVID-19 patients were asymptomatic during the infection and $32 \%$ were asymptomatic at the time of diagnosis in a closed cohort study for COVID-19 outbreak in aircraft carrier[9]. According to the metaanalysis, it is speculated that $33 \%$ of infected people are asymptomatic[10]. It is now considered that antigen test is not suitable for the asymptomatic patients or screening test for the cohort[4, 7]. However, the sensitivity of antigen test has improved recently, and repeated and sequential test could compensate the disadvantage of antigen test for asymptomatic patients.

Another issue is a false positivity of antigen test. As shown in this study, we have to be careful for the interpretation of $100>\mathrm{Ag}>=1.0$. In this setting, we have to make proper diagnosis by additional PCR test in consideration of the clinical information, such as the presence of typical pneumonia, characteristic symptoms like taste disorder and the recent history of contact with COVID-19 patient.

Based upon our foregoing observation, we think that quantitative Ag test for SARS-CoV-2 is useful clinically and should be used more widely and efficiently to make a rapid diagnosis of COVID-19 in the situation with pandemic spread of SARS-CoV-2.

\section{Declarations}

\section{Authors' Contributions}

$\mathrm{MN}$ conceptualized the idea, reviewed the literature and wrote the main draft of the manuscript. RI supported MN. YY, MF, and MD contributed to the assay. KU, NH, RT, and MK collected the clinical data.

Funding The authors have no relevant financial or non-financial interests to disclose 
Compliance of ethical standards

Conflict of Interest The authors have no conflict of interest to declare.

Ethical Approval This study was approved by the institutional review board of Musashino Red Cross Hospital as No 2110.

Informed Consent Informed consent was secured by opt-out method.

\section{References}

1. Zhu N, Zhang D, Wang W, Li X, Yang B, Song J, et al. A Novel Coronavirus from Patients with Pneumonia in China, 2019. N Engl J Med. 2020;382:727-33.

2. Zhou F, Yu T, Du R, Fan G, Liu Y, Liu Z, et al. Clinical course and risk factors for mortality of adult inpatients with COVID-19 in Wuhan, China: a retrospective cohort study. Lancet. 2020;395:1054-62.

3. Organization WH. Weekly epidemiological update on COVID-19-27 April 2021. https://wwwwhoint/publications/m/item/weekly-epidemiological-update-on-covid-19--27-april-2021 2021.

4. Mak GC, Cheng PK, Lau SS, Wong KK, Lau CS, Lam ET, et al. Evaluation of rapid antigen test for detection of SARS-CoV-2 virus. J Clin Virol. 2020;129:104500.

5. Candel FJ, Barreiro P, San Román J, Abanades JC, Barba R, Barberán J, et al. Recommendations for use of antigenic tests in the diagnosis of acute SARS-CoV-2 infection in the second pandemic wave: attitude in different clinical settings. Rev Esp Quimioter. 2020;33:466-84.

6. Menni C, Valdes AM, Freidin MB, Sudre CH, Nguyen LH, Drew DA, et al. Real-time tracking of selfreported symptoms to predict potential COVID-19. Nat Med. 2020;26:1037-40.

7. Hirotsu Y, Maejima M, Shibusawa M, Nagakubo Y, Hosaka K, Amemiya K, et al. Comparison of automated SARS-CoV-2 antigen test for COVID-19 infection with quantitative RT-PCR using 313 nasopharyngeal swabs, including from seven serially followed patients. Int J Infect Dis. 2020;99:397-402.

8. Wölfel R, Corman VM, Guggemos W, Seilmaier M, Zange S, Müller MA, et al. Virological assessment of hospitalized patients with COVID-2019. Nature. 2020;581:465-9.

9. Kasper MR, Geibe JR, Sears CL, Riegodedios AJ, Luse T, Von Thun AM, et al. An Outbreak of Covid-19 on an Aircraft Carrier. N Engl J Med. 2020;383:2417-26. 
10. Oran DP, Topol EJ. The Proportion of SARS-CoV-2 Infections That Are Asymptomatic: A Systematic Review. Ann Intern Med 2021.

\section{Tables}

Please see the supplementary files section to view the tables.

\section{Figures}

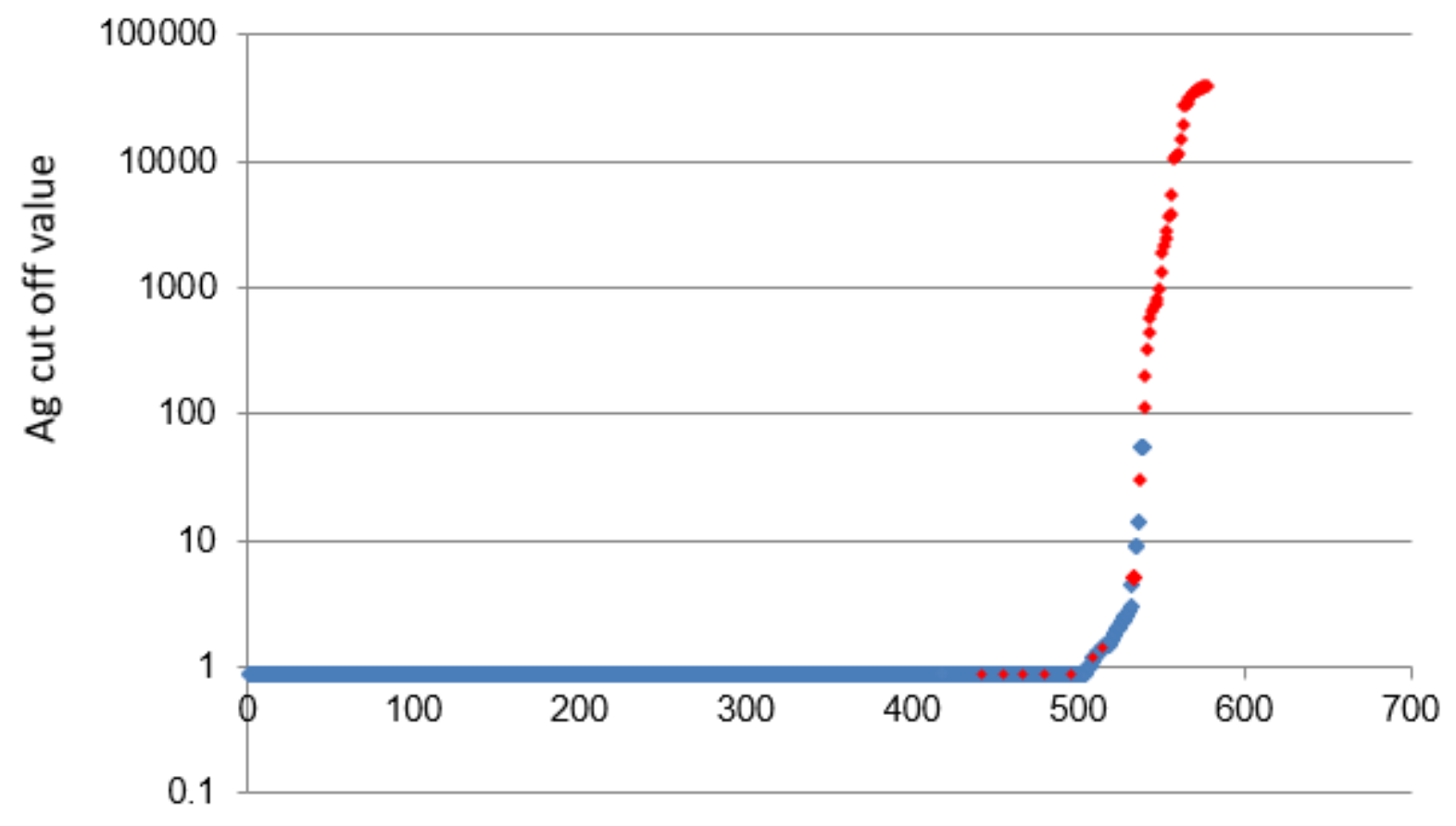

Figure 1

Ag cut off value of each patient Ag cut off value of each patient, in whom Ag and PCR test were conducted at the same time is plotted in ascending order. Red diamond presents a patient with positive PCR test. 


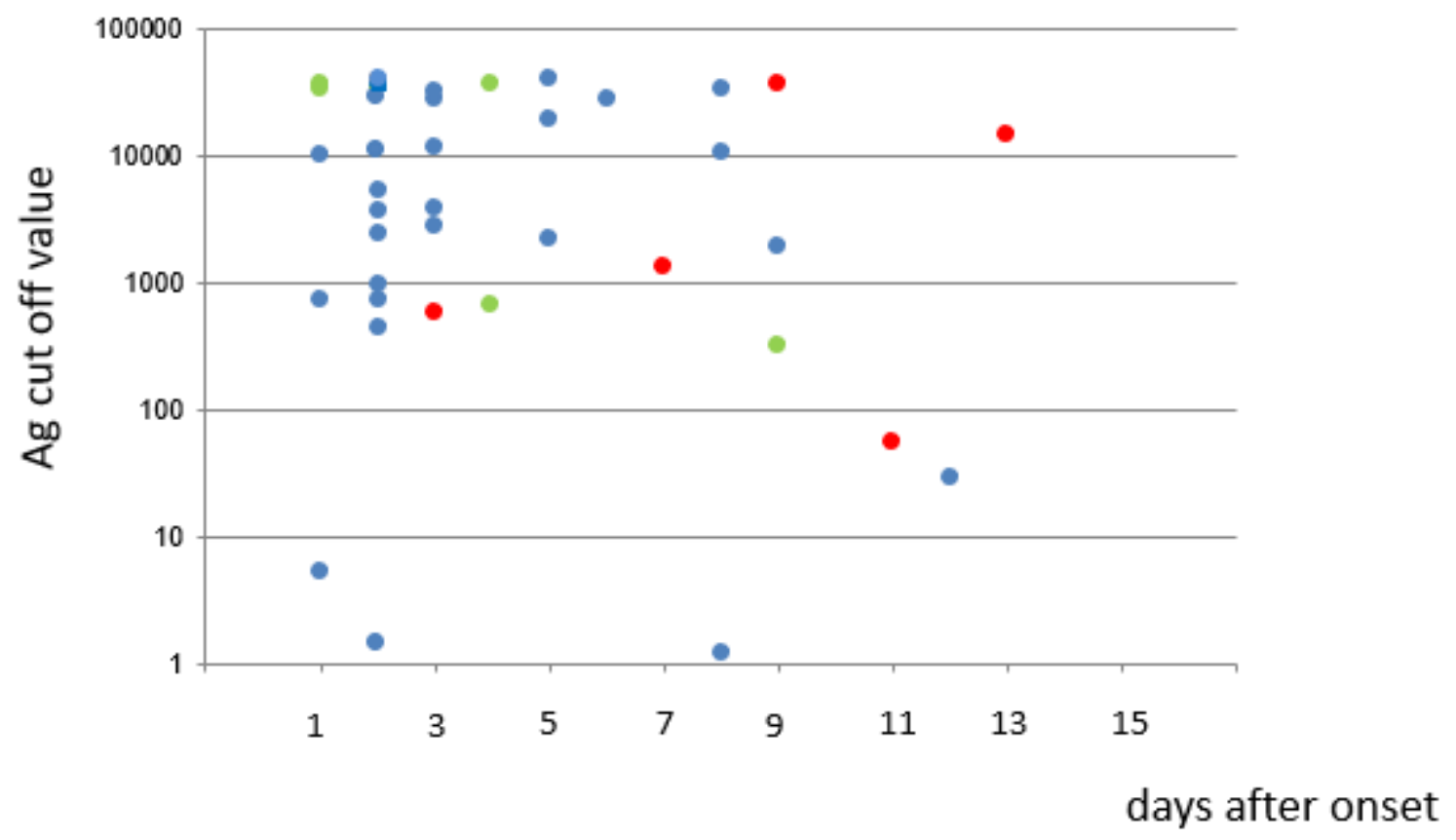

Figure 2

Relation of Ag cut off value and tested date The Ag cut off value and the date when the sample was obtained in the patients with positive PCR test and Ag $>=1.0$ is plotted. Red circle presents the dead patient, and green circle presents the one who received artificial respiratory support and survived.

\section{Supplementary Files}

This is a list of supplementary files associated with this preprint. Click to download.

- 20210429table.pptx 